\title{
An Analysis of Design Factors for Developing Opuntia Humifusa Spines Removal Device
}

\author{
Ik Joo Jang, Yu Shin Ha* \\ Department of Bio-Industrial Machinery Engineering, Kyungpook National University, Daegu, Korea
}

Received: June $10^{\text {th }}, 2013$; Revised: August $6^{\text {th }}, 2013$; Accepted: August $24^{\text {th }}, 2013$

\section{Abstract}

Purpose: Opuntia Humifusa has been used in the food and beauty industry after removing spines and glochids clearly. This study compared the methods used in removing spines and analyzed the design factors for developing a spine removal device. Method: This study compared the spine removal ratios in accordance with the length of brush, water spray pressure, the number of water spray, and the size of Opuntia Humifusa in a rotating brush device and a water spray device. In addition, this study compared the reversal ratios according to the inclination angle of a conveyor, the drop height of Opuntia Humifusa, and the speed of the conveyor to analyze the reversal factors. Results: The spines were not removed clearly in the rotating brush method, and the glochids were nailed deeply. The spine removal ratio was $96.9 \%$ with the water spray pressure of $20 \mathrm{kgf} / \mathrm{cm}^{2}$ and the conveyor speed of $10 \mathrm{~cm} / \mathrm{s}$ in the water spray method. The number of water spray was correlated with the spine removal ratio, and the average spine removal ratio was $95.6 \%$ with three cycles of water spray. The reversal ratio was $97 \%$ with the inclination angle of the conveyor $20^{\circ}$, the drop height of $380 \mathrm{~mm}$, and the conveyor speed of $10 \mathrm{~cm} / \mathrm{s}$. Conclusions: In order to develop a Opuntia humifusa spine removing device, this study compared the rotating brush and water spray methods. As a result, each spine removal performance of the rotating brush and water spray methods was $96.9 \%$ and $95.6 \%$, respectively. Although the performance of the rotating brush method was slightly higher than that of the water spray method, the water spray method was suitable for removing spines from stem because the epidermis of stem was damaged and the glochids were nail deeply in the rotating brush method. Further studies on appropriate arrangement of spray nozzles, maintaining the optimal water spray pressure, the speed and angle control of the feeding conveyor, and devices for inducing the stem to the center will be needed in combining the water spray device and the reversal device.

Keywords: Brush, Opuntia humifusa, Reversal device, Spine remover, Water spray

\section{Introduction}

Opuntia Humifusa is a Korea native cactus which is rich in fiber, calcium, and vitamin $\mathrm{C}$, and its antioxidant effect is higher than Opuntia ficus-indica var saboten (Kwon and Song, 2005; Lee et al, 2004; Park et al, 2005). Its fruits and stems are used in cosmetics, tea, soap, noodle, and rice wine due to the effective ingredient.

Opuntia Humifusa grows up to $30 \mathrm{~cm}$ and has glochids while Opuntia ficus-indica var saboten grows up to 1-2 m

\footnotetext{
*Comesponding author: $\mathrm{Yu}$ Shin $\mathrm{Ha}$

Tel: +82-53-950-5792; Fax: +82-53-950-6780

E-mail: yushin72@knu.ac.kr
}

and has long and thick spines. The stem of one Opuntia Humifusa has 40-60 spine follicles, and $2 \mathrm{~mm}$ long 200 glochids are embedded on the epidermis of one spine follicle (Kim, 2011, Moon, 2003). The spines should be removed to be used in the food and beauty industry; otherwise, it would degrade the taste or hurt the skin (Oh and Lee, 2011). The food processing of Opuntia Humifusa starts with cleaning and freeze-drying, crushing, extracting, mixing, and packaging follow next (Kim and Kim, 2011; Lim, 2012). Therefore, removing spines at the cleaning stage is an essential part in the food processing of Opuntia Humifusa. 
There are several methods to remove spines from Opuntia Humifusa or Opuntia ficus-indica var saboten. Some conventional methods used the friction of rubber (Charles and Felker, 1987) and the plastic brush attached to two rollers (Lydakis et al, 2005; Moon, 2001). Both the methods were applied to removing the spines of Opuntia Humifusa, but the glochids were not removed clearly. According to Kim and Kim (2012), the spines were removed by the friction of the Opuntia Humifusa and the brushes when Opuntia Humifusa and water were placed and agitated in a drum with the brushes. However, this method damaged Opuntia Humifusa and did not remove the glochids. Oh and Lee (2011) sliced the epidermis of Opuntia Humifusa 1.0-1.5 mm using a compressionrotation slice device, but this method degraded the processing yield.

Thus, the spine removing without damage and loss to Opuntia Humifusa is an essential part in the processing of Opuntia Humifusa. This study compared several spine removal methods in order to design an effective device. In addition, this study analyzed the design factors for developing a spine removal device.

\section{Materials and Methods}

This study compared the spine removal ratios in accordance with the length of brush, water spray pressure, the number of water spray, and the size of Opuntia Humifusa using a rotating brush device and a water spray device. In addition, this study compared the reversal ratios according to the inclination angle of a conveyor, the drop height of Opuntia
Humifusa, and the speed of the conveyor to analyze the reversal factors.

\section{Materials}

The frozen stems of Opuntia Humifusa produced at Bian-myun, Uiseong-gun, Gyeongbuk province were used for this study. This study selected and used Opuntia humifusa similar in size because it varies in size from 50 to $100 \mathrm{~mm}$. Different-sized Opuntia humifusa were used because the Opuntia humifusa used for the brush method and the water spray method were produced in different periods. The width of the stem was regarded as the size of Opuntia Humifusa shown in Figure 1(A). Three different sizes of stem, $60 \pm 1.6 \mathrm{~mm}, 80 \pm 0.9 \mathrm{~mm}$, and $85 \pm 1.2 \mathrm{~mm}$, were used with the brush method, and $72 \pm 0.8 \mathrm{~mm}, 83 \pm 1.3 \mathrm{~mm}$, $94 \pm 1.7 \mathrm{~mm}$ were used with the water spray method. Figure 1(B) shows the glochids which were embedded on the epidermis.

\section{Experiments}

\section{Spine removal device by rotating brush}

The rotating brush method removed the spines by rotating a brush on the fixed stem. The device was composed of the rotating brush and a holder which fixed the stem.

The rotating brush was made of polyethylene; two different bristle lengths $20 \mathrm{~mm}$ and $70 \mathrm{~mm}$ were used for this study. The holder should endure the contact force of the brush, and it should be flat to ensure maximum contact area with the curved shape stem. Thus, the holder was made with $2 \mathrm{~mm}$ thick stainless wire by collocating at 20 $\mathrm{mm}$ intervals, and the size of it was $170 \times 320 \mathrm{~mm}$.

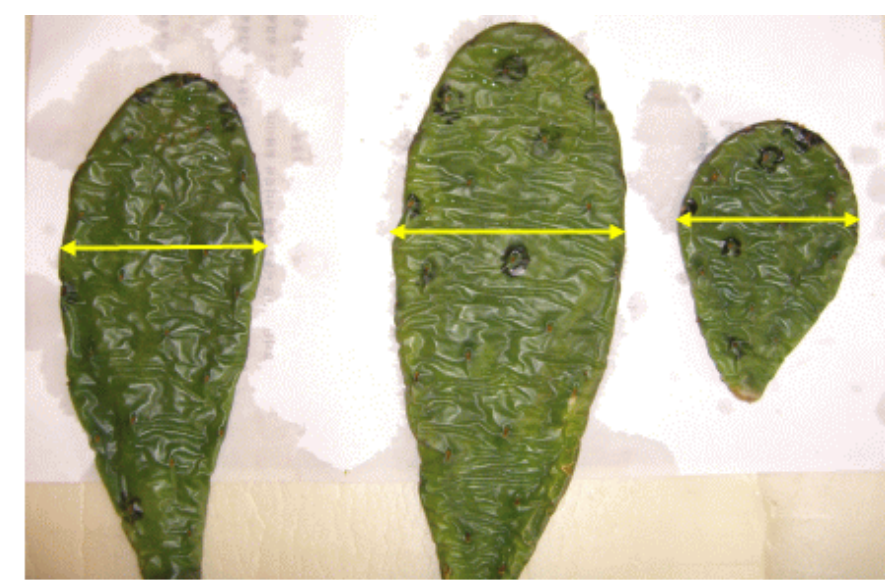

(A)

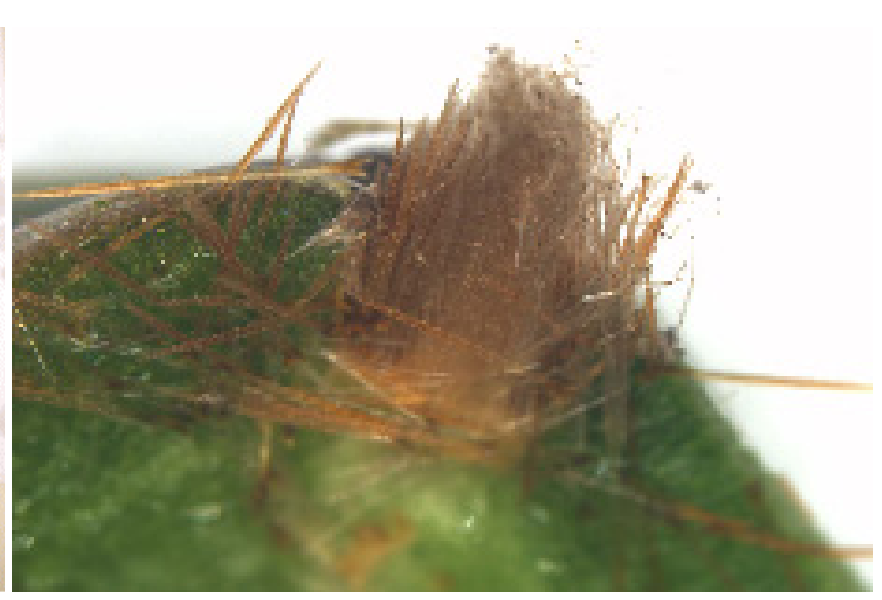

(B)

Figure 1. Three sizes of the stem (A) and glochids in one spine follicle (B). 


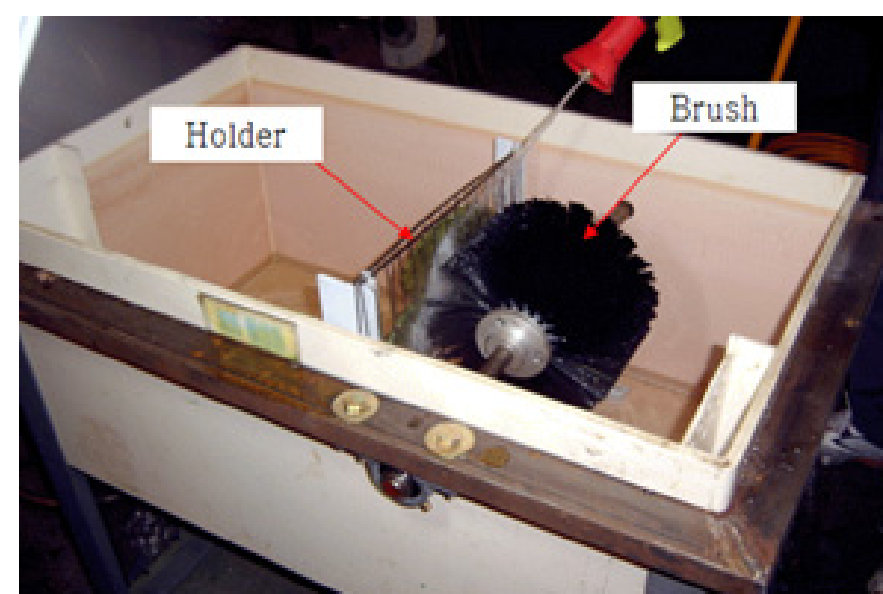

(A)

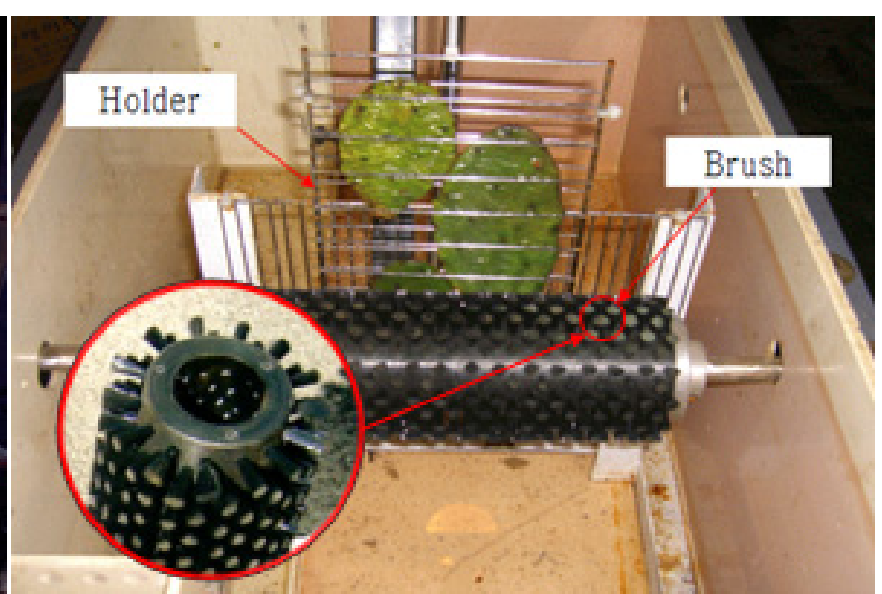

(B)

Figure 2. Rotating brush device with $70 \mathrm{~mm}$ bristle lengths $(\mathrm{A})$ and $20 \mathrm{~mm}(\mathrm{~B})$.

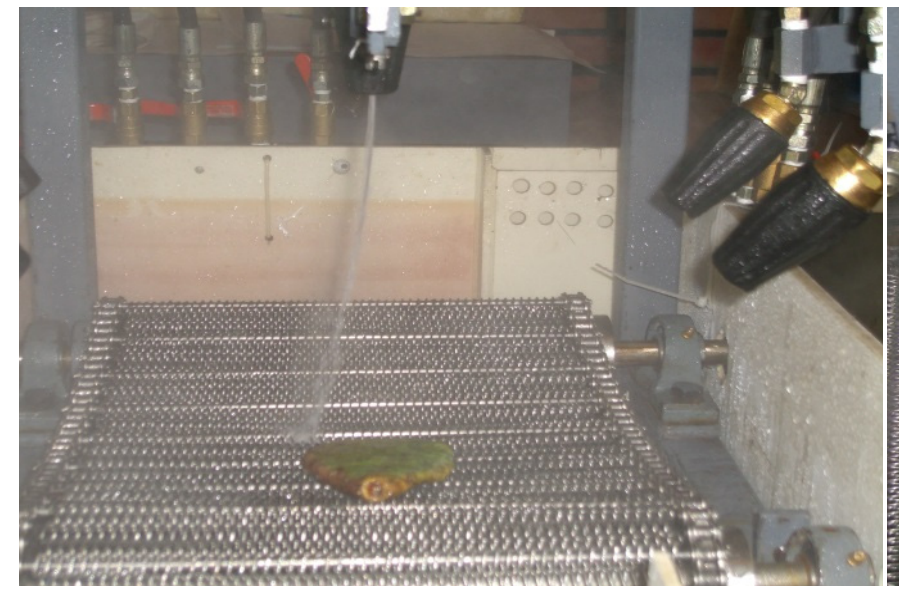

(A)

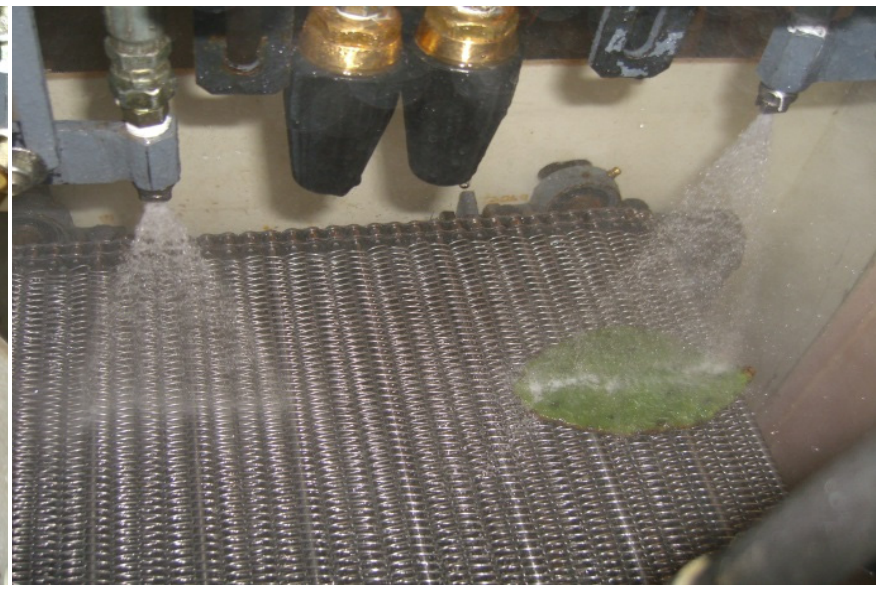

(B)

Figure 3. Rotating nozzle (A) and fan shaped nozzles (B).

\section{Water spray device}

The spines were removed by the water pressure on the stem fixed with various shaped nozzles in the water spray device. In the prior experiment, spines were not removed when using spray type nozzles and removed when using linear type nozzles. However, a linear type nozzle was not effective because the area to remove spines was too small when using it. Linear and rotating nozzle were effective because the area to remove spines became larger when using it.

A ray of water was sprayed at an angle of $20^{\circ}$ in circular motions from the rotating nozzle (Nozzle 7265, Cat Pumps Inc., USA), and the throw distance was set by $200 \mathrm{~mm}$ considering the width of the stem. The device for removing spines on the side was needed because the spines were spread on the side along with the front and the back of the stem. Each two rotating nozzles were arranged on the upper, the left, and the right side of the device, and the spray angle on the side was $45^{\circ}$. Power sprayer (WPS100A, Wondae Precision, Korea) and regulator controlled the pressure of the nozzles.

The stem of Opuntia Humifusa was slipped to the side due to the sprayed water pressure. For this reason, the fan shaped nozzles (VP, DST, Korea) were used for fixing, and they fixed the stem well. The fan shaped nozzles were shown in Figure 3(B), and they were arranged at the same intervals as the rotating nozzles.

A reversal device was needed to remove the spines on the back side after removing them on the front. After the stem was transferred to the conveyor, it was flipped over at the same time with dropping as in Figure 4. The reversal factors were discovered while controlling the angle and 


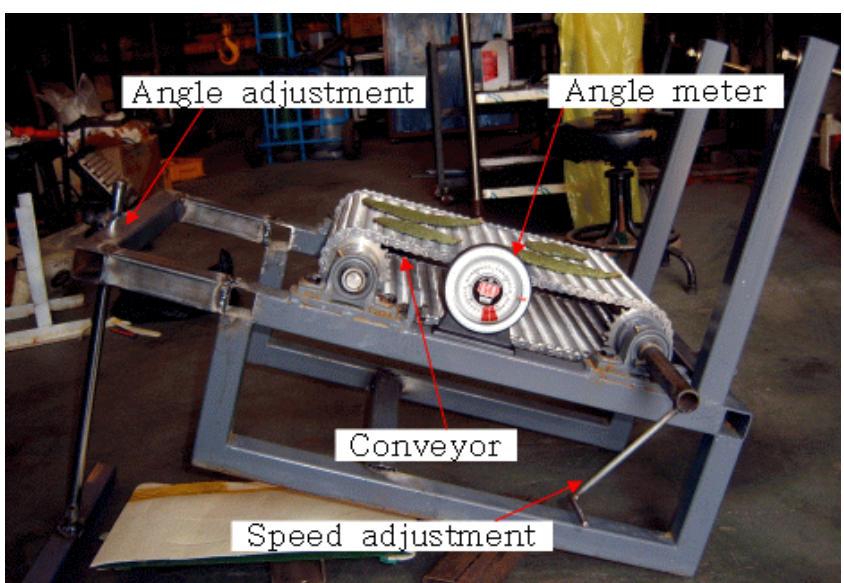

Figure 4. The reversal device.

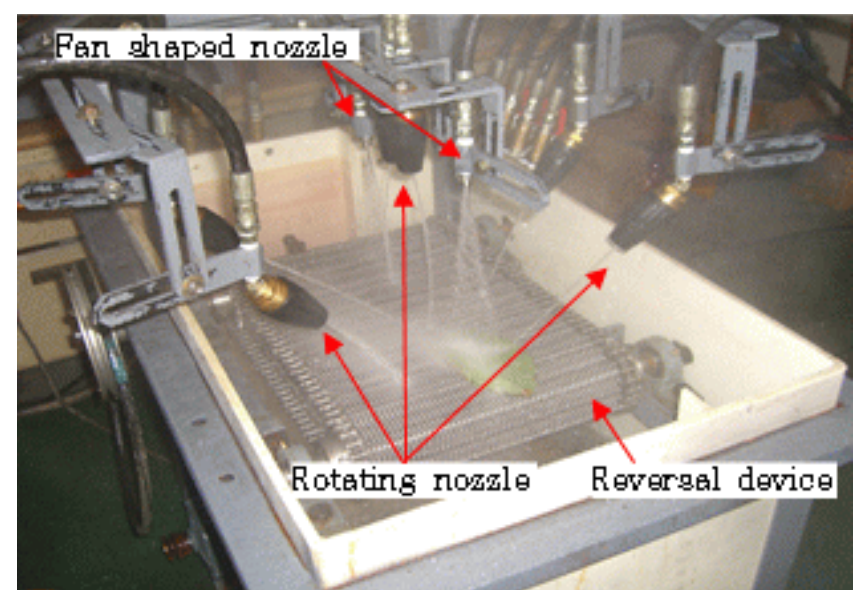

Figure 5. The water spray device with the reversal device.

the speed of the conveyor.

Figure 5 shows the water spray device with the reversal device used for this study.

\section{Method}

\section{Performance test of the spine removal device}

The experiments were conducted to see the performance of the rotating brush method and the water spray method. The performance was represented as a ratio shown in equation (1) by counting the number of the spines before and after the experiment. The removal of the spines was observed by the naked eyes.

$$
T=\frac{B-A}{B} \times 100
$$

where, $\mathrm{T}$ : Spine removal ratio (\%)

$\mathrm{A}$ : the number of the spines after the experiment (ea)
B : the number of the spines before the experiment (ea)

\section{Performance test of the rotating brush}

The experiments were conducted with two different length bristles, $20 \mathrm{~mm}$ and $70 \mathrm{~mm}$, and compared the spine removal performance depending on the bristle lengths. The brushes were directly connected to the rotating motor at 1,710 rpm. The front and the back of the stem were contacted with the brush six times respectively.

\section{Performance test of the water spray device}

Optimal water pressure and the conveyor speed were calculated while varying pressure and speed. With these values, the performance of spine removal of each size of stem and the number of water spray were analyzed.

The experiment was conducted with the water pressure of $15,20,25 \mathrm{kgf} / \mathrm{cm}^{2}$ and the conveyor speed of 5, 10, 15 $\mathrm{cm} / \mathrm{s}$. The stem was placed on the rotating stainless steel chain belt, and the water was sprayed three times on each side of it.

\section{Performance test of the reversal device}

The test was conducted 100 times with the inclination angle of the conveyor $15,20,25^{\circ}$, the drop height of 330 , $380,430 \mathrm{~mm}$, and the conveyor speed of $5,10,15 \mathrm{~cm} / \mathrm{s}$, respectively. The performance was represented as a ratio shown in equation (2) by calculating the number of reversal while dropping and test times.

$$
R=\frac{N r}{N o} \times 100
$$

where, R : Reversal ratio (\%)

$\mathrm{Nr}$ : Number of reversed stem (ea)

No : Number of stem (ea)

\section{Results and Discussion}

\section{Performance result of the spine removal device}

The spine removal ratio by the rotating brush device is shown in Table 1 . The $20 \mathrm{~mm}$ bristle showed $12.4 \%$ removal ratio on the front and back, $2.0 \%$ on the side. The $70 \mathrm{~mm}$ bristle showed $96.9 \%$ on the front and back, but $18.3 \%$ on the side. From the results, the spine removal ratio by the bristle lengths showed that the larger brush was much better than the smaller one and another device for 
Table 1. Spine removal ratio by the rotating brush device (Unit : ea, \%)

\begin{tabular}{|c|c|c|c|c|c|c|}
\hline \multirow{2}{*}{$\begin{array}{l}\text { Brush type } \\
\qquad(\mathrm{mm})\end{array}$} & \multirow{2}{*}{\multicolumn{2}{|c|}{ Thorn bundles }} & \multicolumn{3}{|c|}{ Opuntia humifusa size (mm) } & \multirow{2}{*}{ Average } \\
\hline & & & 60 & 80 & 85 & \\
\hline \multirow{6}{*}{20} & \multirow{2}{*}{ Before } & Back \& forth & 43 & 48 & 45 & 45.3 \\
\hline & & Side & 19 & 17 & 17 & 17.7 \\
\hline & \multirow{2}{*}{ After } & Back \& forth & 38 & 40 & 41 & 39.7 \\
\hline & & Side & 19 & 16 & 17 & 17.3 \\
\hline & \multirow{2}{*}{$\begin{array}{l}\text { Removing } \\
\text { ratio }\end{array}$} & Back \& forth & 11.6 & 16.7 & 8.9 & 12.4 \\
\hline & & Side & 0 & 5.9 & 0 & 2.0 \\
\hline \multirow{6}{*}{70} & \multirow{2}{*}{ Before } & Back \& forth & 43 & 46 & 43 & 44.0 \\
\hline & & Side & 20 & 17 & 18 & 18.3 \\
\hline & \multirow{2}{*}{ After } & Back \& forth & 2 & 0 & 2 & 1.3 \\
\hline & & Side & 14 & 17 & 14 & 15.0 \\
\hline & \multirow{2}{*}{$\begin{array}{l}\text { Removing } \\
\text { ratio }\end{array}$} & Back \& forth & 95.3 & 100 & 95.3 & 96.9 \\
\hline & & Side & 30 & 0 & 22.2 & 17.4 \\
\hline
\end{tabular}

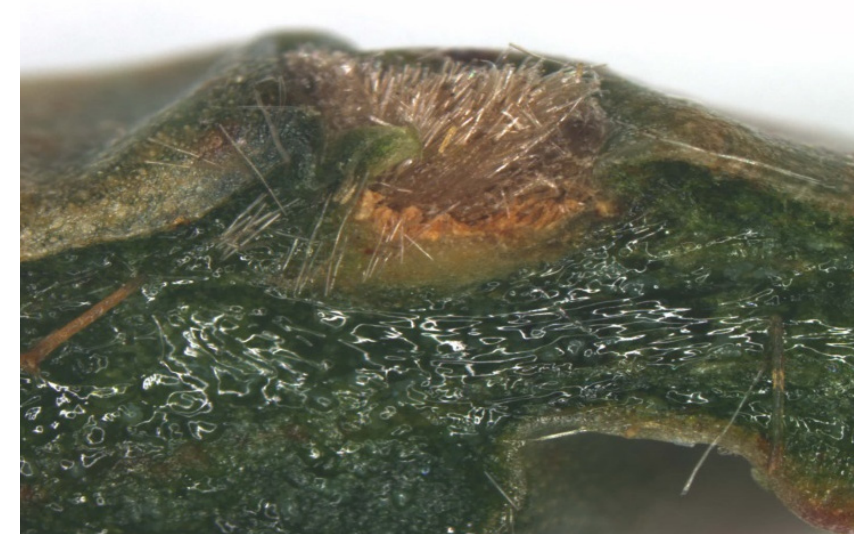

Figure 6. Shot of the spine-removed stem taken with an electron microscope.

removing spines on the side was needed.

However, a shot of the spine-removed stem taken with an electron microscope showed that glochids were embedded on the epidermis though the spines on the surface were removed. When the brush was rotated more to remove the glochids, the epidermis of the stem was damaged and the glochids were nail deeply. Therefore, the rotating method was not suitable for removing spines from the stem.

\section{Performance result of the water spray device}

Performance result in accordance with the water spray pressure and the conveyor speed

The performance result in accordance with the water spray pressure and the conveyor speed is shown in Table 2. The performance results showed the spine removal
Table 2. Spine removal ratio in accordance with the water spray pressure and the conveyor speed (Unit : \%)

\begin{tabular}{|c|c|c|c|c|}
\hline \multirow{2}{*}{$\begin{array}{c}\text { Water injection } \\
\text { pressure } \\
\left(\mathrm{kgf} / \mathrm{cm}^{2}\right)\end{array}$} & \multicolumn{3}{|c|}{ Conveyor speed $(\mathrm{cm} / \mathrm{s})$} & \multirow[b]{2}{*}{ Remarks } \\
\hline & 5 & 10 & 15 & \\
\hline 15 & 88 & 80 & 76 & - \\
\hline 20 & 98 & 97 & 92 & - \\
\hline 25 & - & - & - & Damage \\
\hline
\end{tabular}

ratio of $98 \%$ with the water spray pressure of $20 \mathrm{kgf} / \mathrm{cm}^{2}$ and the conveyor speed of $5 \mathrm{~cm} / \mathrm{s}$. But in terms of work efficiency, the conveyor speed of $10 \mathrm{~cm} / \mathrm{s}$ was considered the best though the ratio was $97 \%$. The water spray pressure of $25 \mathrm{kgf} / \mathrm{cm}^{2}$ damaged the stem, so it was not suitable for removing the spines.

Since optimal water spray pressure was $20 \mathrm{kgf} / \mathrm{cm}^{2}$, there should be regulator to maintain the water spray pressure $20 \mathrm{kgf} / \mathrm{cm}^{2}$.

Performance result in accordance with the number of water spray and the stem size

Table 3 shows the spine removal ratio in accordance with the number of water spray and the stem size. When the spines and water spray direction were at right angles to each other, they were removed $100 \%$. However, the spines on the curvy stems were not completely removed because the spines and water spray direction were not at right angles to each other. There was a tendency that small size of the stem had high spine removal ratio, but it showed no significant difference $(P>0.05)$. However, the 
Table 3. Thorn removing ratio by the size and number of injection(Unit : ea, \%)

\begin{tabular}{|c|c|c|c|c|c|}
\hline \multirow{2}{*}{$\begin{array}{l}\text { Number of } \\
\text { cycles }^{\text {a) }}\end{array}$} & \multirow{2}{*}{ Thorn bundles } & \multicolumn{3}{|c|}{ Size of stem $(\mathrm{mm})$} & \multirow{2}{*}{ Average } \\
\hline & & 72 & 83 & 94 & \\
\hline \multirow{3}{*}{1} & Before & 21 & 22 & 25 & 22.7 \\
\hline & After & 8 & 9 & 8 & 8.3 \\
\hline & Removing ratio & 61.9 & 59.1 & 68 & 63.0 \\
\hline \multirow{3}{*}{2} & Before & 21 & 22 & 25 & 22.7 \\
\hline & After & 3 & 4 & 5 & 4.0 \\
\hline & Removing ratio & 85.7 & 81.8 & 80 & 82.5 \\
\hline \multirow{3}{*}{3} & Before & 21 & 22 & 25 & 22.7 \\
\hline & After & 0 & 1 & 2 & 1.0 \\
\hline & Removing ratio & 100 & 95.5 & 92 & 95.8 \\
\hline
\end{tabular}

a) Significantly different parameter between number of cycles

\begin{tabular}{|c|c|c|c|c|}
\hline \multirow{2}{*}{$\begin{array}{c}\text { Angle }^{a)} \\
\left({ }^{\circ}\right)\end{array}$} & \multirow{2}{*}{$\begin{array}{l}\text { Drop height } \\
\quad(\mathrm{mm})\end{array}$} & \multicolumn{3}{|c|}{ Conveyor speed $(\mathrm{cm} / \mathrm{s})$} \\
\hline & & 5 & 10 & 15 \\
\hline \multirow{3}{*}{15} & 330 & 76 & 79 & 77 \\
\hline & 380 & 78 & 81 & 79 \\
\hline & 430 & 75 & 77 & 76 \\
\hline \multirow{3}{*}{20} & 330 & 91 & 94 & 92 \\
\hline & 380 & 96 & 97 & 95 \\
\hline & 430 & 90 & 93 & 91 \\
\hline \multirow{3}{*}{25} & 330 & 73 & 75 & 73 \\
\hline & 380 & 74 & 76 & 73 \\
\hline & 430 & 72 & 73 & 70 \\
\hline
\end{tabular}

a) Significantly different parameter between angle

number of water spray was correlated with the spine removal ratio $(\mathrm{P}<0.001)$. Three cycles of water spray showed the best result (95.8\%), but it damaged the surface of the stem. Thus, no more than three cycles of water spray should be arranged when developing the process with spines removal devices.

\section{Performance result of the reversal device}

Table 4 shows the reversal efficiency of the stem with the reversal device. The reversal ratio was high with $97 \%$ when the inclination angle was $20^{\circ}$ with drop height of $380 \mathrm{~mm}$ and speed of $10 \mathrm{~cm} / \mathrm{s}$.

Reversal ratio was not correlated with the conveyor speed and the drop height $(\mathrm{P}>0.05)$, but the conveyor speed of $10 \mathrm{~cm} / \mathrm{s}$ was suitable considering the optimal speed of the spine removal ratio. Inclination angle of $20^{\circ}$ was suitable $(\mathrm{P}<0.001)$, but the stems were slipped back with the angle of $25^{\circ}$. In addition, the stem arranged in the middle of the conveyor was not landed at the same position after dropping and reversing. Considering the water spray range and angle, the stems should be placed on the middle of the conveyor to remove the spines effectively. Therefore, a device for inducing the stem to the center was needed to remove the spines on the back side of the stems.

\section{Conclusions}

Opuntia Humifusa has excellent effective components, so it is used in the food and beauty industry after spines and glochids were removed clearly. This study compared the methods used in removing spines and glochids. The results of the analysis about the design factors for developing a spine removal device are as follows:

(1) The spine removal device with $70 \mathrm{~mm}$ long bristle showed $96.9 \%$ on the front and back, but $18.3 \%$ on the side. This study found that the brush method was not appropriate because it did not remove glochids at all. Furthermore, it needs another device for removing spines on the side.

(2) The performance result showed the spine removal ratio of $96.9 \%$ with the water spray pressure of 20 $\mathrm{kgf} / \mathrm{cm}^{2}$ and the conveyor speed of $10 \mathrm{~cm} / \mathrm{s}$. However, when the brush was rotated more to remove the glochids, the epidermis of the stem was damaged and the glochids were nail deeply. Therefore, the rotating method was not suitable for removing spines from the stem.

(3) Performance result of the spine removal ratio in accordance with the number of water spray and the stem size showed that the number of water spray was correlated with the spine removal ratio. Three cycles of water spray showed the best result 95.8\%.

(4) The reversal ratio of the stem was high with $97 \%$ when the inclination angle was $20^{\circ}$ with drop height of $380 \mathrm{~mm}$ and speed of $10 \mathrm{~cm} / \mathrm{s}$.

The water spray method was effective in removing spines of Opuntia Humifusa. Further studies on appropriate arrangement of spray nozzles, maintaining the optimal water spray pressure, the speed and angle control of the feeding conveyor, and devices for inducing the stem to the 
center after reversing will be needed in combining the water spray device and the reversal device.

\section{Conflict of Interest}

The authors have no conflicting financial or other interests.

\section{Acknowledgement}

This research was supported by Kyungpook National University Research Fund, 2012.

\section{References}

Charles, E. R and P. Felker. 1987. The Prickly-pears (Opuntia spp., Cactaceae): A Source of human and animal food in Semiarid Regions. Economic Botany 41(3):433-445.

Kim, D. S. 2011. Oputia humifusa cultivation and storage technology. Seoul: Pubple (in Korean).

Kim, J. K and M. G. Kim. 2012. Slice of Multipurpose Slice Cactus. Korean Patent Publication No.1020120078227 (In Korean).

Kwon, D. K and Y. J. Song. 2005. Effect of Opuntia humifusa supplementation on endurance exercise performance in rats fed a high-fat diet. The Korean Journal of
Exercise Nutrition 9(2):183-188 (in Korean).

Lee K. S., M. G. Kim and K. Y. Lee. 2004. Antimicrobial effect of the extracts of cactus Chounnyouncho (Opuntia humifusa) against food borne pathogens. Journal of the Korean Society Food Science and Nutrition. 33(8):1268-1272 (in Korean).

Lee, M. I and J. S. Oh. 2011. A manufacturing process of a slice pack for beauty of a raw cactus stem (leaf). Korean Patent Publication No.1020110030047 (In Korean).

Lim, 2012. Development of small packaging juice powder with Opuntia spp. Goyang Agriculture Technology Center (in Korean).

Lydakis, D., N. Pompodakisa, E. Markelloub and S.M. Lionakisa. 2005. Storage response of cactus pear fruit following hot water brushing. Postharvest Biology and Technology 38(2):145-151.

Moon, Y. I. 2000. Device for removing a thorn of a falm cactus fruit. Korean Patent Publication No.1020010090192 (In Korean).

Moon, Y. I. 2003. Studies on cultural practices, composition and functional effect of OPuntia ficus-indica var. savoten. PhD diss. Cheju: Cheju national University, Department of Horticulture (in Korean).

Park, M. K., Y. J. Lee and E. S. Kang. 2005. Hepatoprotective effect of Cheonnyuncho (Opuntia humifusa) extract in rats treated carbon tetrachloride. Korean Journal of Food Science and Technology 37(5):822-826 (in Korean). 